\title{
Subsídios ao processo de seleção de sistemas de tratamento de esgotos - Proposição de estrutura de preferência para Análise Multicriterial
}

\author{
Subsidies to selection of sewage treatment systems - Preference structure proposition for \\ Multicriteria Analysis \\ Subvenciones para el proceso de selección de sistemas de tratamiento de aguas residuales - \\ Proposición de una estructura de preferencias para el Análisis Multicriterio
}

Recebido: 19/08/2021 | Revisado: 24/08/2021 | Aceito: 25/08/2021 | Publicado: 26/08/2021

Adriana de Oliveira Pereira dos Reis
ORCID: https://orcid.org/0000-0001-9834-751X
Instituto Federal de Educação, Ciência e Tecnologia do Espírito Santo, Brasil
E-mail: aopreis @ gmail.com
José Antonio Tosta dos Reis
ORCID: https://orcid.org/0000-0001-9916-1469
Universidade Federal do Espírito Santo, Brasil
E-mail: jatreis@ gmail.com

\begin{abstract}
Resumo
Este trabalho teve por objetivo estabelecer, a partir de Pesquisa Qualitativa, estrutura de preferência aplicável ao processo de seleção de sistemas de tratamento de esgotos, processo que usualmente envolve conjunto diversificado de critérios e que abrange aspectos ambientais, operacionais, econômicos e sociais. Foram considerados dezenove critérios distribuídos entre quatro subsistemas sociotécnicos. O Subsistema Ambiental apresentou-se como o mais relevante para a seleção dos sistemas de tratamento de esgotos. Dentre os critérios analisados, eficiência de remoção de matéria orgânica, eficiência de remoção de coliformes e requisitos de energia foram considerados os mais importantes para os especialistas consultados. Interferência decorrente da geração de aerossóis e Possibilidade de atração de insetos, critérios associados ao Subsistema Social, apresentam-se como os de menor importância para o referido processo de seleção.
\end{abstract}

Palavras-chave: Pesquisa qualitativa; Grupo focado; Tratamento de esgotos; Critérios de seleção.

\begin{abstract}
This work's main goal was to establish, from qualitative research, criteria and weigth applicable to the selection process of wastewater treatment systems, a process that usually involves a many criteria and differents environmental, operational, economic and social aspects. Nineteen criteria were considered, distributed among four socio-technical subsystems. The Environmental Subsystem was the most relevant for selection process. Removal organic matter efficiency, removal coliforms efficiency and energy demand were considered the most important criteria by specialists. Interference of aerosols generation and the possibility of insect attraction, criteria associated to the Social Subsystem, were considered as the least important criteria.
\end{abstract}

Keywords: Qualitative research; Focus group; Wastewater treatment; Selection criteria.

\section{Resumen}

Este trabajo tuvo como objetivo establecer, a partir de la Investigación Cualitativa, una estructura preferente aplicable al proceso de selección de los sistemas de tratamiento de aguas residuales, proceso que suele implicar un conjunto diversificado de criterios y que engloba aspectos ambientales, operativos, económicos y sociales. Se consideraron diecinueve criterios distribuidos entre diferentes subsistemas sociotécnicos. El subsistema Medioambiental fue el más relevante para la selección de sistemas de tratamiento de aguas residuales. Entre los criterios analizados, la eficiencia de remoción de materia orgánica, la eficiencia de remoción de coliformes y los requerimientos energéticos fueron considerados los más importantes para los expertos consultados. La interferencia derivada de la Generación de Aerosoles y la Posibilidad de atraer insectos, criterios asociados al subsistema Social, se presentan como los menos importantes para el referido proceso de selección.

Palabras clave: Investigación cualitativa; Grupos focales; Tratamiento de aguas residuales; Criterios de selección.

\section{Introdução}

No Brasil, o diagnóstico das condições de esgotamento sanitário das sedes municipais indicou que, de toda a carga orgânica produzida no País (aproximadamente 9,1 mil toneladas diárias de DBO), apenas 39\% é removida com a infraestrutura 
de tratamento de esgoto existente. Neste contexto, cerca de 5,5 mil toneladas diárias de DBO alcançam os cursos d'água sem tratamento adequado (ANA, 2017).

Considerando-se que, em função do tipo de tecnologia selecionada, os custos de investimentos e operação de sistemas de tratamento de esgotos podem oscilar substancialmente (Tchobanoglous \& Burton, 2003; Von Sperling, 2005; Jordão \& Pessôa, 2005), o processo de escolha das plantas de tratamento deve ser conduzido de forma cuidadosa.

Diversos fatores devem ser considerados na escolha de um determinado sistema, sobretudo restrições ambientais como vazão de efluentes brutos, características de qualidade do afluente, disponibilidade de terreno, condições climáticas, eficiência do sistema, processamento do lodo e dos demais resíduos gerados no processo de tratamento, incluindo-se demanda por energia e de material humano (Tchobanoglous \& Burton, 2003).

Os critérios considerados no processo de escolha do sistema de tratamento usualmente são diferenciados entre países desenvolvidos e em desenvolvimento. Em países desenvolvidos, a eficiência, a confiabilidade, a disposição do lodo e a disponibilidade de área são fatores considerados críticos no processo de escolha do sistema de tratamento de esgotos, enquanto que para os países em desenvolvimento, aspectos como custos de construção e de operação, simplicidade e sustentabilidade são aspectos críticos para a tomada de decisão (Von Sperling, 1996; Yu et al., 1997; Von Sperling, 2005).

Segundo Oliveira (2004), quando consideradas as crescentes pressões associadas à manutenção e recuperação da qualidade dos ambientes naturais, a melhor alternativa de tratamento de esgotos é aquela que garante eficiência ecológica e econômica. Quando são consideradas as cobranças para manutenção e recuperação da qualidade dos corpos de água, o melhor sistema de tratamento de esgoto é aquele que confere eficiência ecológica e econômica. Desta forma, a seleção do melhor sistema de tratamento de esgoto baseia-se na minimização do consumo de energia, da geração de resíduos e dos custos de implantação, operação e manutenção, com garantia de remoção dos poluentes, além do cumprimento dos padrões de qualidade ambiental.

Leoneti (2009), por sua vez, considerou os custos de operação e manutenção (O\&M) e os investimentos iniciais da implantação da planta de tratamento de esgoto como os critérios mais importantes para processo de seleção de sistemas de tratamento de esgotos. Karimi et al (2011) sugeriram confiabilidade e flexibilidade do sistema, custos de implantação, custos de O\&M, quantidade de lodo gerado, disposição do lodo, impactos ambientais, capacidade de assimilação de cargas afluentes, demanda por área, clima, além dos padrões de qualidade ambiental de alguns parâmetros físico-químicos como odor, oxigênio dissolvido, sólidos suspensos, nitrogênio e fósforo, como critérios relevantes para a seleção de sistemas de tratamento de esgotos.

Neste contexto, torna-se evidente que o processo de seleção de sistemas de tratamento de esgotos deve ser conduzido a partir de abordagem multicriterial, onde existem tanto aspectos qualitativos como quantitativos que devem ser avaliados conjuntamente, quando da tomada de decisão acerca do sistema de tratamento dos esgotos mais viável para uma determinada condição de produção de efluentes e sua disposição final (Golmohammadi \& Mellat-Parast, 2012; Kalbar et al., 2012).

Esta pesquisa tem por objetivo estabelecer, a partir do emprego de Pesquisa Qualitativa, estrutura de preferência que ofereça suporte ao processo de seleção de sistemas de tratamento de esgotos por meio do emprego de métodos multicritério de seleção, como os métodos da família ELECTRE, da família PROMETHEE e a Teoria da Utilidade Multiatributo (MAUT), dentre outros.

\section{Metodologia}

\subsection{Pesquisa Qualitativa}

O presente trabalho envolveu a condução de Pesquisa Qualitativa (Koche, 2011; Estrela, 2018; Pereira et al., 2018), levada a termo com auxílio da conformação de Grupos Focados, atividade por meio da qual se buscou identificar a taxonomia 
dos critérios sociotécnicos que permitam adequadamente orientar o processo de seleção de sistemas de tratamento de esgoto no âmbito de uma bacia hidrográfica.

Critérios aplicáveis ao processo de seleção de sistemas de tratamento de esgotos e os subsistemas sociotécnicos (Subsistemas Ambiental, Tecnológico, Operação e Social) aos quais estariam associados, identificados a partir de revisão de literatura, passaram por processo de validação. Esta etapa do trabalho permitiu a classificação dos diferentes critérios de seleção nos diferentes subsistemas sociotécnicos considerados. Após o processo de validação, reuniões em três grupos focados (grupos com composição e condução detalhados em item subsequente) foram realizadas. A partir da condução dos grupos focados foi possível estabelecer, por meio da obtenção dos pesos associados aos critérios e aos subsistemas sociotécnicos, a estrutura de preferência aplicável ao processo de seleção de tecnologias de tratamento de esgotos no âmbito de uma bacia hidrográfica.

\subsection{Validação dos critérios e dos subsistemas sociotécnicos}

Os subsistemas sociotécnicos (Ambiental, Tecnológico, Operação e Social) e os critérios de seleção passaram por um processo de validação por especialistas. Os especialistas que realizaram a validação possuem expertise nas áreas que se relacionam com o processo de seleção, concepção, projeto e operação de sistemas de tratamento de esgotos. O processo de validação foi conduzido em duas etapas:

- Primeira etapa - validação quanto à classificação dos critérios nos subsistemas ambiental, tecnológico, operação e social.

- Segunda etapa - validação dos critérios a serem considerados na seleção de tecnologias de tratamento de esgotos.

Os especialistas, na primeira etapa do processo de validação, verificaram se os critérios estavam adequadamente associados a um subsistema sociotécnico, conforme proposição inicial estabelecida a partir de revisão de literatura. Na segunda etapa do processo de validação, os especialistas avaliaram se os critérios inicialmente reunidos eram relevantes ou não e, assim, se deveriam ser excluídos ou conservados para um processo de seleção de sistemas de tratamento de esgotos. Uma proposição foi considerada válida quando $60 \%$ dos especialistas, no mínimo, apresentaram-se favoráveis à proposição.

Uma síntese dos participantes do processo de validação, incluindo-se formação acadêmica, tempo de experiência e vinculação profissional é apresentada por meio do Quadro 1. 
Quadro 1 - Especialistas participantes do processo de validação.

\begin{tabular}{|c|c|c|c|c|c|}
\hline 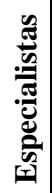 & 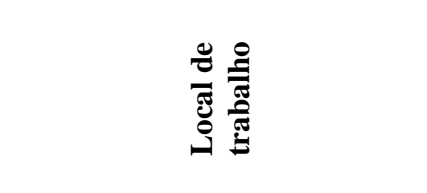 & 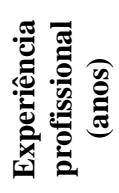 & & 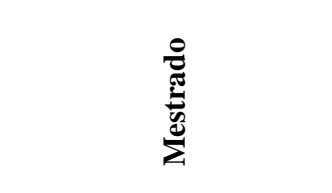 & $\begin{array}{l}\stackrel{0}{\frac{\pi}{\pi}} \\
\stackrel{0}{0} \\
\stackrel{0}{0}\end{array}$ \\
\hline 1 & Instituição de Ensino e Pesquisa & 13 & Engenharia Civil & $\begin{array}{c}\text { Engenharia Hidráulica e } \\
\text { Saneamento }\end{array}$ & $\begin{array}{c}\text { Engenharia Hidráulica e } \\
\text { Saneamento }\end{array}$ \\
\hline 2 & Instituição de Ensino e Pesquisa & 10 & Engenharia Química & $\begin{array}{c}\text { Engenharia Hidráulica e } \\
\text { Saneamento }\end{array}$ & $\begin{array}{c}\text { Engenharia Hidráulica e } \\
\text { Saneamento }\end{array}$ \\
\hline 3 & Instituição de Ensino e Pesquisa & 40 & Engenharia Civil & $\begin{array}{c}\text { Engenharia Hidráulica e } \\
\text { Saneamento }\end{array}$ & $\begin{array}{c}\text { Engenharia de Recursos } \\
\text { Hídricos }\end{array}$ \\
\hline 4 & Instituição de Ensino e Pesquisa & 22 & Engenharia Civil & Engenharia Ambiental & $\begin{array}{c}\text { Engenharia Hidráulica e } \\
\text { Saneamento }\end{array}$ \\
\hline 5 & Instituição de Ensino e Pesquisa & 32 & Engenharia Civil & Hidráulica e Saneamento & $\begin{array}{c}\text { Engenharia Hidráulica e } \\
\text { Saneamento }\end{array}$ \\
\hline 6 & Empresa de Saneamento & 9 & $\begin{array}{l}\text { Tecnologia em } \\
\text { Saneamento } \\
\text { Ambiental }\end{array}$ & $\begin{array}{l}\text { Gestão e Regulação de } \\
\text { Recursos Hídricos }\end{array}$ & - \\
\hline 7 & Empresa de Saneamento & 12 & Ciências Biológicas & Engenharia Ambiental & - \\
\hline 8 & Empresa de Saneamento & 11 & Química Industrial & - & - \\
\hline 9 & Empresa de Saneamento & 12 & Engenharia Civil & Engenharia Ambiental & - \\
\hline 10 & Órgão Gestor ou Regulador & 12 & Engenharia Civil & Engenharia Ambiental & - \\
\hline 11 & Órgão Gestor ou Regulador & 2 & Engenharia Química & - & - \\
\hline 12 & Órgão Gestor ou Regulador & 9 & Engenharia Química & - & - \\
\hline 13 & Órgão Gestor ou Regulador & 11 & Engenharia Agrícola & Engenharia Agrícola & - \\
\hline
\end{tabular}

Fonte: Autores (2021).

\subsection{Planejamento e condução dos Grupos Focados}

Neste trabalho, o Grupo Focado foi empregado como técnica de Pesquisa Qualitativa. Segundo Lervorino e Pelicioni (2001), Ribeiro e Newmann (2012), o Grupo Focado constitui técnica de pesquisa que pode ser empregada quando se deseja compreender percepções e atitudes acerca de um fato, prática, produto ou serviço. Segundo os referidos autores, o grupo focado consiste, essencialmente, na interação entre os participantes e o pesquisador, objetivando-se reunir dados por meio de discussão focada em tópicos específicos e diretivos. O Grupo Focado tem constituído técnica de pesquisa utilizada em pesquisas desenvolvidas em diferentes áreas do conhecimento, como ilustram os trabalhos de Carlini-Cotrim (1996), Kidd e Parshall (2000), Conroy (2003), Yoshikawa et al. (2008), Runeson e Höst (2009), Säynäjoki et al. (2014), De Wolf et al. (2017).

No planejamento dos grupos focados foram definidos o objetivo da reunião, os participantes, o local e as questões a serem discutidas (Ribeiro \& Newmann, 2003). A autora desta pesquisa atuou como moderador. O objetivo das entrevistas conduzidas por grupo focado foi realizar uma análise taxonômica dos critérios sociotécnicos selecionados após a etapa de 
validação. Os participantes integraram três grupos focados, assim definidos:

- Grupo Focado 1 (GF1) - Academia: grupo formado por acadêmicos e pós-graduandos, com experiência na área de recursos hídricos e saneamento ambiental (universidades e institutos federais);

- Grupo Focado 2 (GF2) - Tratamento: grupo formado por profissionais da área de saneamento, com experiência na área de tratamento de esgotos e recursos hídricos (Concessionária responsável pela operação de sistemas de tratamento de esgotos) e;

- Grupo Focado 3 (GF3) - Regulação: grupo formado por profissionais vinculados ao órgão regulador de recursos hídricos (agência reguladora dos recursos hídricos).

O GF1 foi conduzido em reunião da qual participaram três especialistas, sendo um professor de Universidade Federal, um professor de Instituto Federal e um doutorando de Engenharia Ambiental, este último proprietário de empresa de consultoria na área de tratamento de esgotos. Os professores são doutores em Engenharia Civil, com ênfase em Hidráulica e Saneamento, com experiência comprovada em recursos hídricos e saneamento. Já do GF2 participaram cinco especialistas da área de saneamento, com experiência em operação de sistemas de tratamento de esgoto e/ou processos de outorga e licenciamento de estações de tratamento de esgotos. O GF3 foi constituído por três especialistas da área de regulação em recursos hídricos, com atuação em outorga e/ou experiência em licenciamento ambiental.

Em relação às questões utilizadas nos grupos focados, buscando-se facilitar os trabalhos desta fase da pesquisa, foram utilizados formulários com os critérios já validados. O moderador utilizou, para condução dos grupos focados, notebook, projetor e gravador para registro dos comentários dos participantes nas sessões. As sessões tiveram duração de, no máximo, duas horas e foram conduzidas pelo moderador. Nas reuniões não foram utilizadas gravação em vídeo para evitar um eventual incômodo dos participantes e prejuízo na obtenção de informações.

Os participantes dos grupos focados atribuíram pesos aos critérios e subsistemas sociotécnicos, utilizando uma escala de 1 a 10. Em função do julgamento do nível de importância de cada um dos critérios e dos subsistemas sociotécnicos, cada participante atribuiu peso 1 para o critério e/ou subsistema com menor importância dentre os demais dados e; peso 10 para aquele critério e/ou subsistema com maior importância em relação aos outros critérios e/ou subsistemas.

Como resultado da pesquisa qualitativa foram obtidos os pesos de importância associados aos critérios de seleção de tratamento de esgotos e aos subsistemas sociotécnicos. Por meio da média aritmética dos pesos obtidos nos três grupos focados, obteve-se a estrutura de preferência para os subsistemas e critérios sociotécnicos.

\section{Resultados e Discussão}

Conforme previamente indicado, processo de validação ocorreu em duas etapas. Na primeira etapa da validação os especialistas deveriam indicar se concordavam com uma proposta preliminar de alocação dos critérios nos subsistemas Ambiental, Tecnológico, Operação e Social. Caso não concordassem com a distribuição proposta, poderiam alocar os critérios objetos de análise em qualquer outro sistema sociotécnicos. Num segundo momento da validação os especialistas poderiam sugerir a inclusão de novos critérios de seleção, bem como sugerir a supressão de critérios que fossem considerados desnecessários, ainda que previamente associados a algum subsistema. Os resultados decorrentes da primeira etapa da validação estão reunidos na Tabela 1.

Na Tabela 1 está apresentado o percentual de concordância dos especialistas quanto a alocação de cada critério nos subsistemas sociotécnicos com os quais foram inicialmente associados. Os menores percentuais de concordâncias (67\%) foram registrados para a alocação dos critérios a) Quantidade de lodo a ser tratado e b) Quantidade de lodo a ser disposto no subsistema tecnológico. As propostas de alocação dos demais critérios por subsistema sociotécnicos receberam aprovação de, no mínimo, $75 \%$ dos especialistas consultados. É relevante observar que todos os especialistas consultados concordaram com 
os critérios propostos para a conformação do Subsistema Ambiental.

Alguns dos especialistas consultados sugeriram a inserção de um mesmo critério em mais de um subsistema, sob a justificativa de transversalidade do critério assinalado. Nesta pesquisa optou-se por enquadrar os critérios em um único subsistema sociotécnico, tornando mais objetiva a condução dos grupos focados, principalmente quando da atribuição dos pesos aos critérios e subsistemas.

Tabela 1 - Resultados da primeira etapa do processo de validação - Alocação dos critérios nos subsistemas sociotécnicos.

\begin{tabular}{|c|c|c|}
\hline \multicolumn{2}{|c|}{ Proposição de classificação dos critérios nos subsistemas sociotécnicos } & \multirow{2}{*}{$\begin{array}{c}\text { \% Concordância entre } \\
\text { especialistas }\end{array}$} \\
\hline Subsistemas Sociotécnicos & Critérios de seleção propostos & \\
\hline \multirow{4}{*}{ Ambiental } & Eficiência de Remoção de Matéria Orgânica & $100 \%$ \\
\hline & Eficiência de Remoção de Nitrogênio & $100 \%$ \\
\hline & Eficiência de Remoção de Fósforo & $100 \%$ \\
\hline & Eficiência de Remoção de Coliformes & $100 \%$ \\
\hline \multirow{6}{*}{ Tecnológico } & Requisitos de Energia & $83 \%$ \\
\hline & Demanda por Área de Implantação & $100 \%$ \\
\hline & Custos de Implantação & $92 \%$ \\
\hline & Custos de Operação & $100 \%$ \\
\hline & Quantidade de Lodo a ser tratado & $67 \%$ \\
\hline & Quantidade de Lodo a ser disposto & $67 \%$ \\
\hline \multirow{5}{*}{ Operação } & Confiabilidade do sistema & $83 \%$ \\
\hline & Simplicidade Operacional & $75 \%$ \\
\hline & Capacidade de Resistência a Variações de Vazão & $100 \%$ \\
\hline & Capacidade de Resistência a Variações das Características do Afluente & $75 \%$ \\
\hline & Capacidade de Resistência a Constituintes Tóxicos & $75 \%$ \\
\hline \multirow{4}{*}{ Social } & Interferência decorrente da geração de Odor & $100 \%$ \\
\hline & Interferência decorrente da Geração de Ruído & $92 \%$ \\
\hline & Interferência decorrente da Geração de Aerossóis & $92 \%$ \\
\hline & Possibilidade de atração de Insetos & $92 \%$ \\
\hline
\end{tabular}

Fonte: Autores (2021).

Outros critérios foram sugeridos de maneira isolada ou por parcela minoritária dos especialistas consultados, como a) possibilidade de reaproveitamento de subprodutos e do efluente tratado, b) possibilidade de cogeração de energia, c) capacidade de autodepuração do corpo receptor dos efluentes tratados, d) vazão remanescente no ponto de lançamento para diluição e depuração de efluentes tratados, segundo critério de outorga do órgão de controle de recursos hídricos, e) características dos corpos d'água a jusante do ponto de lançamento (ambientes lênticos, lóticos ou intermediários), f) características relacionadas com meio ambiente da área de implantação da ETE, g) localização da ETE em relação a área urbanizada e h) menor consumo de insumos químicos (por exemplo: no acondicionamento do afluente e tratamento do lodo).

A determinação dos pesos de importância para os critérios e subsistemas sociotécnicos - que conformam a estrutura de preferência empregada para a seleção dos sistemas de tratamento de esgotos - foram definidos após condução dos grupos focados. Os pesos utilizados no processo de seleção, tanto para os critérios quanto para os subsistemas sociotécnicos, foram obtidos a partir da média aritmética dos valores obtidos nos grupos focados. Os pesos dos subsistemas sociotécnicos e dos critérios de seleção, por Grupo Focado, estão reunidos nas tabelas 2 e 3, respectivamente. A Tabela 4 apresenta, de maneira 
sintética, a estrutura de preferência sugerida para o processo de seleção de sistemas de tratamento de esgotos.

Tabela 2 - Pesos atribuídos aos subsistemas sociotécnicos obtidos por meio dos Grupos Focados

\begin{tabular}{cccc}
\hline Subsistema Sociotécnico & GF 1 (Academia) & GF 2 (Tratamento) & GF 3 (Regulação) \\
\hline Ambiental & 10 & 9 & 10 \\
\hline Tecnológico & 8 & 9 & 8 \\
\hline Operação & 6 & 8 & 7 \\
\hline Social & 3 & 7 & 8 \\
\hline
\end{tabular}

Fonte: Autores (2021).

Tabela 3 - Pesos atribuído aos critérios de seleção obtidos por meio dos Grupos Focados.

\begin{tabular}{|c|c|c|c|}
\hline Critérios de seleção validados & $\begin{array}{c}\text { GF 1 } \\
\text { (Academia) }\end{array}$ & $\begin{array}{c}\text { GF 2 } \\
\text { (Tratamento) }\end{array}$ & $\begin{array}{c}\text { GF 3 } \\
\text { (Regulação) }\end{array}$ \\
\hline Eficiência de Remoção de Matéria Orgânica & 10 & 10 & 10 \\
\hline Eficiência de Remoção de Nitrogênio & 8 & 6 & 7 \\
\hline Eficiência de Remoção de Fósforo & 8 & 6 & 8 \\
\hline Eficiência de Remoção de Coliformes & 10 & 8 & 8 \\
\hline Requisitos de Energia & 8 & 8 & 8 \\
\hline Demanda por Área de Implantação & 5 & 7 & 7 \\
\hline Custos de Implantação & 8 & 7 & 6 \\
\hline Custos de Operação & 9 & 8 & 6 \\
\hline Quantidade de Lodo a ser tratado & 7 & 5 & 5 \\
\hline Quantidade de Lodo a ser disposto & 7 & 8 & 5 \\
\hline Confiabilidade do sistema & 3 & 9 & 6 \\
\hline Simplicidade Operacional & 6 & 7 & 5 \\
\hline Capacidade de Resistência a Variações de Vazão & 5 & 7 & 6 \\
\hline Capacidade de Resistência a Variações das Características do Afluente & 5 & 7 & 6 \\
\hline Capacidade de Resistência a Constituintes Tóxicos & 3 & 4 & 3 \\
\hline Interferência decorrente da geração de Odor & 6 & 8 & 6 \\
\hline Interferência decorrente da Geração de Ruído & 2 & 7 & 5 \\
\hline Interferência decorrente da Geração de Aerossóis & 2 & 4 & 2 \\
\hline Possibilidade de atração de Insetos & 2 & 3 & 2 \\
\hline
\end{tabular}

Fonte: Autores (2021). 
Tabela 4 - Estruturas de preferência utilizada para o processo de seleção de sistemas de tratamento de esgotos.

\begin{tabular}{|c|c|c|c|}
\hline $\begin{array}{l}\text { Subsistemas } \\
\text { Sociotécnicos }\end{array}$ & $\begin{array}{c}\text { Peso dos } \\
\text { Subsistemas }\end{array}$ & Critérios de seleção & $\begin{array}{l}\text { Peso dos } \\
\text { Critérios }\end{array}$ \\
\hline \multirow{4}{*}{ Ambiental } & \multirow{4}{*}{9,6} & Eficiência de Remoção de Matéria Orgânica & 10,0 \\
\hline & & Eficiência de Remoção de Nitrogênio & 7,0 \\
\hline & & Eficiência de Remoção de Fósforo & 7,3 \\
\hline & & Eficiência de Remoção de Coliformes & 8,7 \\
\hline \multirow{6}{*}{ Tecnológico } & \multirow{6}{*}{8,3} & Requisitos de Energia & 8,0 \\
\hline & & Demanda por Área de Implantação & 6,3 \\
\hline & & Custos de Implantação & 7,0 \\
\hline & & Custos de Operação & 7,7 \\
\hline & & Quantidade de Lodo a ser tratado & 5,7 \\
\hline & & Quantidade de Lodo a ser disposto & 6,7 \\
\hline \multirow{5}{*}{ Operação } & \multirow{5}{*}{7,1} & Confiabilidade do sistema & 6,0 \\
\hline & & Simplicidade Operacional & 6,0 \\
\hline & & Capacidade de Resistência a Variações de Vazão & 6,0 \\
\hline & & $\begin{array}{c}\text { Capacidade de Resistência a Variações das Características do } \\
\text { Afluente }\end{array}$ & 6,0 \\
\hline & & Capacidade de Resistência a Constituintes Tóxicos & 3,3 \\
\hline \multirow{4}{*}{ Social } & \multirow{4}{*}{6,0} & Interferência decorrente da geração de Odor & 6,7 \\
\hline & & Interferência decorrente da Geração de Ruído & 4,7 \\
\hline & & Interferência decorrente da Geração de Aerossóis & 2,7 \\
\hline & & Possibilidade de atração de Insetos & 2,3 \\
\hline
\end{tabular}

Fonte: Autores (2021).

Da simples inspeção da Tabela 2 é possível observar que, dentre os subsistemas sociotécnicos avaliados, o subsistema Ambiental tem prevalência sob os demais, independentemente do grupo focado analisado (para o segundo grupo focado, os subsistemas Ambiental e Tecnológico assumiram igual importância). De maneira similar, para o conjunto dos grupos focados, os aspectos associados ao Subsistema Social foram os considerados menos importantes para o processo de seleção de sistemas de tratamento de esgotos. No entanto, é relevante observar que no primeiro GF1, o Subsistema Social apresentou importância relativa muito menor que aquela que lhe foi atribuída nos demais grupos focados. Participantes do GF1 argumentaram que, ao se garantir tecnologias com maiores eficiências, consequentemente seriam assegurados sistemas que possivelmente trariam menores impactos sociais. Já o GF2 justificou a atribuição de valores relativamente maiores para o Subsistema Social, função do número de reclamações e exigências da população nos canais de atendimento da concessionária. Os subsistemas Tecnológico e Operação, nesta ordem, completaram o ordenamento dos subsistemas sociotécnicos.

A análise dos pesos atribuídos aos critérios de seleção (pesos reunidos na Tabela 3) permite observar que a eficiência de remoção de matéria orgânica é considerada como o critério de maior importância para o processo de seleção de sistemas de tratamento de esgotos, constituindo o único critério que recebeu peso 10 (máximo valor atribuível para um critério) em todos os grupos focados. O GF1, no entanto, foi o único grupo que atribuiu peso 10 (dez) para um outro critério de seleção - a eficiência de remoção de coliformes - apontando-o com mesmo nível de importância que a eficiência de remoção de matéria 
orgânica. Para o GF1 não há como dissociar a importância desses dois critérios no tratamento de esgotos. Para a média dos grupos focados, a eficiência de remoção de matéria orgânica, eficiência de remoção de coliformes e requisitos de energia, nesta ordem, constituíram os critérios de maior importância para o processo de seleção, todos com pesos iguais ou superiores a 8,0 (oito).

Alguns critérios apresentaram valores diferenciados entre os grupos, dentre os quais merecem destaque a confiabilidade do sistema e a interferência decorrente da geração de ruído. O GF1 argumentou que, ambos os critérios seriam automaticamente atendidos pela escolha de tecnologias que garantissem o nível de eficiência de remoção de DBO e coliformes em valores mais representativos. Já o GF3 entendeu que a interferência decorrente da geração de odor teria maior influência comparativamente à geração de ruído. No conjunto dos grupos focados, os critérios considerados menos importantes foram a capacidade de resistência a constituintes tóxicos, a interferência decorrente da geração de aerossóis e a possibilidade de atração de insetos, todos com pesos médios inferiores a 4,0 (quatro).

\section{Considerações Finais}

Esta pesquisa estabeleceu estrutura de preferência aplicável ao processo de seleção de sistemas de tratamento de esgotos com auxílio de métodos multicriteriais. Foram avaliados e hierarquizados quatro subsistemas sociotécnicos e dezenove critérios de seleção distribuídos entre os referidos subsistemas.

O Subsistema Ambiental foi considerando o mais relevante para a seleção de sistemas de tratamento de esgoto, independentemente do grupo de especialistas consultados. Os aspectos associados ao Subsistema Social, por sua vez, foram indicados como os de menor importância.

Dentre os critérios de seleção analisados, a Eficiência de remoção de matéria orgânica foi considerada como o mais importante por todos os especialistas que participaram da pesquisa, independentemente de sua formação ou vinculação profissional. A Eficiência de remoção de coliformes e os Requisitos de energia também constituíram critérios que, no conjunto de especialistas consultados, apresentam-se como relevantes para o processo de seleção de sistemas de tratamento de esgotos. Os critérios Interferência decorrente da geração de aerossóis e Possibilidade de atração de insetos, ambos integrantes do Subsistema Social, apresentam-se como os de menor importância para o referido processo de seleção.

Recomenda-se que trabalhos futuros sejam conduzidos com ampliação dos Grupos Focados, considerando diversificação da formação acadêmica e da atuação profissional dos seus integrantes.

\section{Agradecimentos}

À Coordenação de Aperfeiçoamento de Pessoal de Nível Superior (Capes), por bolsa de estudos concedida ao primeiro autor desta pesquisa.

\section{Referências}

ANA - Agência Nacional das Águas. (2017). Atlas Esgotos: despoluição de bacias hidrográficas. ANA.

Carlini-Cotrim, B. (1996). Potencialidades da técnica qualitativa grupo focal em investigações sobre abuso de substâncias. Revista de Saúde Pública, 30, 285293. https://www.scielo.br/j/rsp/a/Gn7VGm9Wkj3YhTBKb5DjmDs/?lang=pt\&format=pdf.

Conroy, S. A. (2003). A pathway for interpretive phenomenology. International Journal of Qualitative Methods, 2(3), 36-62. https://doi.org/10.1177/160940690300200304.

De Wolf, C., Pomponi, F. \& Moncaster, A. (2017). Measuring embodied carbon dioxide equivalent of buildings: A review and critique of current industry practice. Energy and Buildings, 140, 68-80. https://doi.org/10.1016/j.enbuild.2017.01.075.

Estrela, C. (2018). Metodologia Científica: Ciência, Ensino, Pesquisa. Editora Artes Médicas. 
Golmohammadi, D. \& Mellat-Parast, M. (2012). Developing a grey-based decision-making model for supplier selection. International Journal of Production Economics, 137(2), 191-200. https://doi.org/10.1016/j.ijpe.2012.01.025.

Jordão, E. P \& Pessôa, C. A. (2005). Tratamento de esgotos domésticos. ABES.

Kalbar, P. P., Karmakar, S. \& Asolekar, S. R. (2012) Selection of an appropriate wastewater treatment technology: A scenario-based multiple-attribute decision-making approach. Journal of environmental management, 113, 158-169. https://doi.org/10.1016/j.jenvman.2012.08.025.

Karimi, A. R., Mehrdadi, N., Hashemian, S. J., Nabi Bidhendi, G. R. \& Tavakkoli Moghaddam, R. (2011). Selection of wastewater treatment process based on the analytical hierarchy process and fuzzy analytical hierarchy process methods. International Journal of Environmental Science \& Technology, 8(2), 267-280. https://link.springer.com/content/pdf/10.1007/BF03326215.pdf.

Kidd, P. S. \& Parshall, M. B. (2000). Getting the focus and the group: enhancing analytical rigor in focus group research. Qualitative health research, 10(3), 293-308. https://doi.org/10.1177/104973200129118453.

Koche, J. C. (2011). Fundamentos de metodologia científica. Vozes.

Leoneti, A. B. (2009). Avaliação de modelo de tomada de decisão para escolha de sistema de tratamento de esgoto sanitário (Doctoral dissertation, Universidade de São Paulo). https://www.teses.usp.br/teses/disponiveis/96/96132/tde-06052009-144711/en.php.

Lervolino, S.A. \& Pelicioni, M. C. F. (2001). A utilização do grupo focal como metodologia qualitativa na promoção da saúde. Revista da Escola de Enfermagem da USP, 35(.2), 115-121. https://www.scielo.br/j/reeusp/a/kFzCC9Dfbfv7WzPNQbJZVmF/?format=pdf\&lang=pt.

Oliveira, S. V. W. B. D. (2004). Modelo para tomada de decisão na escolha de sistema de tratamento de esgoto sanitário (Doctoral dissertation, Universidade de São Paulo). https://www.teses.usp.br/teses/disponiveis/12/12139/tde-19092006-125541/en.php.

Pereira, A. S., Shitsuka, D. M., Parreira, F. J., \& Shitsuka, R. (2018). Metodologia da pesquisa científica. UFSM.

Ribeiro, J. \& Newmann, C. R. (2012). Estudos qualitativos com o apoio de Grupos Focados. XIII Semana de Engenharia de Produção Sul-americana. Gramado.

Ribeiro, J., \& Newmann, C. (2003). Planejamento e condução de grupos focados. Grupos focados: teoria e aplicações. FEEng - UFRGS.

Runeson, P. \& Höst, M. (2009). Guidelines for conducting and reporting case study research in software engineering. Empirical software engineering, 14(2), 131-164. https://doi.org/10.1007/s10664-008-9102-8.

Säynäjoki, E., Heinonen, J. \& Junnila, S. (2014). The power of urban planning on environmental sustainability: a focus group study in Finland. Sustainability, 6(10), 6622-6643. https://doi.org/10.3390/su6106622.

Tchobanoglous, G. \& Burton, F. L. (1991). Wastewater Engineering - Treatment, Disposal and Reuse. McGraw Hill.

Von Sperling, M. (1996). Princípios do tratamento biológico de águas residuárias - Lagoas de Estabilização. DESA - UFMG.

Von Sperling, M. (2005). Princípios do tratamento biológico de águas residuárias - Introdução à qualidade das águas e ao tratamento de esgotos. DESA UFMG.

Yoshikawa, H., Weisner, T. S., Kalil, A. \& Way, N. (2008). Mixing qualitative and quantitative research in developmental science: uses and methodological choices. Developmental Psychology, 4(2), 344, 2008. https://doi.org/10.1037/0012-1649.44.2.344.

Yu, H., Tay, J. \& Wilson, F. (1997). A sustainable municipal wastewater treatment process for tropical and subtropical regions in developing countries. Water science and technology, 35(9), 191-198. https://doi.org/10.1016/S0273-1223(97)00197-2. 